\title{
Indirect signs of the Peccei-Quinn mechanism
}

\author{
Jordy de Vries, ${ }^{1,2}$ Patrick Draper, ${ }^{1,3}$ Kaori Fuyuto, ${ }^{1}$ Jonathan Kozaczuk, ${ }^{1,3}$ and Dave Sutherland ${ }^{4}$ \\ ${ }^{1}$ Amherst Center for Fundamental Interactions, Department of Physics, University of Massachusetts, \\ Amherst, Massachusetts 01003, USA \\ ${ }^{2}$ RIKEN BNL Research Center, Brookhaven National Laboratory, Upton, New York 11973-5000, USA \\ ${ }^{3}$ Department of Physics, University of Illinois, Urbana, Illinois 61801, USA \\ ${ }^{4}$ Department of Physics, University of California, Santa Barbara, California 93106, USA
}

(Received 6 November 2018; published 31 January 2019)

\begin{abstract}
In the standard model, the renormalization of the QCD vacuum angle $\theta$ is extremely tiny, and small $\theta$ is technically natural. In the general standard model effective field theory (SMEFT), however, $\Delta \theta$ is quadratically divergent, reflecting the fact that new sources of hadronic $C P$-violation typically produce $\mathcal{O}(1)$ threshold corrections to $\theta$. The observation of such $C P$-violating interactions would therefore be in tension with solutions to the strong $C P$ problem in which $\theta=0$ is an ultraviolet boundary condition, pointing to the Peccei-Quinn mechanism as the explanation for why $\theta$ is small in the infrared. We study the quadratic divergences in $\theta$ arising from dimension-6 SMEFT operators and discuss the discovery prospects for these operators at electric dipole moment experiments, the LHC, and future proton-proton colliders.
\end{abstract}

DOI: $10.1103 /$ PhysRevD.99.015042

\section{INTRODUCTION}

The absence of an electric dipole moment (EDM) for the neutron strongly constrains the $C P$-violating $\mathrm{QCD}$ vacuum angle to be tiny, $\theta \lesssim 10^{-10}[1-4]$. Yet $C P$ is evidently not an exact symmetry: it is explicitly broken in the weak interactions, and must be broken further to provide the baryon asymmetry. Finding an explanation for the smallness of $\theta$ in the presence of other sources of $C P$ violation (CPV) constitutes the strong $C P$ problem.

Unlike the other fine-tuning problems of the standard model (SM), nature as we know it seems largely insensitive to $\theta$ : little about nuclear physics [5] would change if $\theta$ were of order $10^{-3}$, for example. For this reason it is widely believed that strong $C P$ must have a dynamical, rather than anthropic, explanation, and we will make this assumption here. ${ }^{1}$

Most of the proposed solutions to strong $C P$ fall into two categories. In ultraviolet (UV) solutions, $\theta=0$ is a consequence of a microscopic symmetry, typically $C P$ (Nelson-Barr models) [8-11] or P [12-16]. At some

\footnotetext{
${ }^{1}$ For a recent attempt to connected the smallness of $\theta$ with anthropics via scanning of the cosmological constant, see $[6,7]$ for further discussion. Also, anthropic effects could conceivably play some role in, e.g., the precise realization of the Peccei-Quinn mechanism, since axions influence the cosmological history.

Published by the American Physical Society under the terms of the Creative Commons Attribution 4.0 International license. Further distribution of this work must maintain attribution to the author(s) and the published article's title, journal citation, and DOI. Funded by SCOAP .
}

intermediate scale, this symmetry is spontaneously broken, and the effects of symmetry breaking are communicated to the SM in ways that are engineered to preserve $\theta=0$. Perhaps the most compelling aspect of these models is that they take advantage of an odd property of the SM: $\theta \approx 0$ is technically natural, in the sense that radiative corrections to $\theta$ are extremely small. The first infinite and finite renormalizations induced by the Cabibbo-Kobayashi-Maskawa (CKM) phase arise at 7 and 4 loop order, respectively [17-19], and the latter has been estimated to generate $\theta \sim 10^{-17}$ [19]. Thus, if $\theta=0$ can be preserved through the scale of spontaneous symmetry breaking, and the Effective Field Theory (EFT) at lower scales is just the SM, $\theta$ will remain sufficiently small at low scales.

In infrared (IR) solutions, $\theta$ can be absorbed into a redefinition of light fields, and the strong interactions are $C P$-conserving down to arbitrarily low scales. Under such circumstances it can also be proven that QCD does not spontaneously break $C P$ [20], so the strong $C P$ problem is solved. One possibility, the massless up quark, is now strongly disfavored by lattice data [21]. ${ }^{2}$ The remaining viable IR solution is the Peccei-Quinn (PQ) mechanism $[23,24]$. In this case the light field that absorbs $\theta$ is the axion $a[25,26]$ and its potential is given by the $\theta$-dependence of the QCD vacuum energy, which is minimized at $\theta=0$ by the same theorem [20].

\footnotetext{
${ }^{2}$ Although further study could still be of interest, and also provide an interesting probe of small instantons in QCD [22].
} 
Curiously, and for different reasons, neither the UV nor the IR solutions discussed above are entirely robust. A wellknown example is that UV sources of explicit PQ-breaking lead to extra terms in the axion potential, stabilizing it in the wrong place and leading to a nonzero effective $\theta$ [27-29]. To preserve $\theta \lesssim 10^{-10}$, the coefficients of Planck-scale operators must be suppressed up to high dimension $(d \sim 12$ for PQ-breaking scales of order $10^{12} \mathrm{GeV}$.) In other words, the PQ symmetry must be of very high quality, broken only by the QCD anomaly to great precision. This problem might be avoided with a string axion [30], but generally with other costs, including a nonsupersymmetric moduli problem [31]. On the other hand, the most fragile component of UV solutions is the small renormalization of $\theta$ below the scale of spontaneous $\mathrm{P} / C P$-breaking. If the EFT is not the $\mathrm{SM}$, there can be new couplings that eventually feed the CPV spurion into $\theta$. For example, in supersymmetry, if CPV is not strongly sequestered from the SUSYbreaking sector, invariant phases in the soft parameters generate various threshold corrections to $\theta$ much larger than $10^{-10}$ [32-34]. Likewise, in models with extra stronglycoupled gauge sectors coupled to the SM, new vacuum angles can shift $\theta$ at the confinement scale of the new sector $[35,36]$. These corrections generically spoil UV solutions to strong $C P .^{3}$

Experimentally, the best hope for resolving the strong $C P$ problem is the detection of an axion component of dark matter [41-44]. In contrast, UV solutions to strong $C P$ do not make generic predictions for lower scales other than that $\theta$ should be small. However, the fragility discussed above provides another handle: UV mechanisms can still be excluded by discovering any new physics that induces quantum corrections to $\theta$ greater than $10^{-10}$. Such a discovery would provide an upper bound on the scale at which strong $C P$ is solved and strong indirect evidence for the PQ mechanism.

We will discuss quantum contributions to $\theta$ and associated experimental signatures in the context of the standard model effective theory (SMEFT). If integrating out heavy fields generates CPV SMEFT operators involving quarks and gluons, it will also typically produce threshold corrections to $\theta$. These corrections are not calculable in the low energy theory, but traces of them remain, including quadratically divergent corrections to $\theta$ involving the effective operators. These quadratic divergences have the same interpretation as that of the Higgs mass in the SM: they reflect strong sensitivity of the renormalizable coupling to UV physics. In the case of $\theta$ the sensitivity is

\footnotetext{
${ }^{3}$ Heavy axion solutions (e.g., [37,38]) based on mirror $\mathbb{Z}_{2}$ symmetries instead of $\mathrm{P} / C P$ are subject to similar issues [39]. It has been argued that in cosmological supersymmetry breaking models, an $R$-axion may receive exotic contributions to its mass from interactions with the horizon that preserve the solution to strong $C P$ [40]. If this is the case, our arguments do not apply to such models.
}

cutoff-independent, $\Lambda^{2} / \Lambda^{2}$, and the relevant UV physics includes any new sources of CPV coupled to quarks or gluons. Thus, evidence for these operators sharpens the unnaturalness of small $\theta$ and strongly disfavors the possibility of natural UV solutions. We note that similar observations can be used to constrain neutrino magnetic moments based on naturalness of the neutrino masses $[45,46]$.

CPV SMEFT operators can be searched for at colliders and in nuclear, atomic, and molecular EDM experiments. With EDMs it is a complicated matter to extract precise values for the many Wilson coefficients involved. However, for our purposes, we need only to rule out a bare value of $\theta$ as the only source of CPV in the strong interactions at low energies. In general this requires two measurements, at least one of which exhibits a signal. Collider probes of CPV operators, on the other hand, are not "contaminated" by $\theta$ in this way, so in principle only one measurement is required. In most cases collider sensitivity to single operators falls short of EDMs, but are still interesting, particularly for operators involving third generation quarks.

This study is organized as follows. In Sec. II we compute the complete quadratic divergence in $\theta$ from dimension- 6 SMEFT operators. In Sec. III we discuss the ability of nucleon, nuclear, and diamagnetic EDM measurements to discriminate the $\theta$-only hypothesis from $\theta+$ SMEFT. In Sec. IV we estimate the collider sensitivity to CPV SMEFT operators, surveying the existing literature and comparing to the EDM reach. The top chromo-EDM is of particular interest, and we study the potential for the high-luminosity $14 \mathrm{TeV}$ LHC and future 27 and $100 \mathrm{TeV}$ colliders to detect the top cEDM in simple angular observables, where it can be distinguished from a $C P$-conserving magnetic moment. In Sec. $V$ we summarize and conclude.

\section{QUADRATIC DIVERGENCES AND $\overline{\boldsymbol{\theta}}$}

We wish to consider the combined $C P$ violating effects of the standard model and some extra beyond-the-standardmodel physics, encoded in the Lagrangian ${ }^{4}$

$$
\mathcal{L}=\mathcal{L}_{\mathrm{SM}}+\mathcal{L}_{\mathrm{BSM}}
$$

$\mathcal{L}_{\mathrm{SM}}$ contains, in addition to the CKM phase, one physical strong $C P$ phase given by the invariant combination of $\theta$ and the phases in the Yukawa couplings,

$$
\bar{\theta}=\theta+\arg \operatorname{det} Y_{u}+\arg \operatorname{det} Y_{d} .
$$

$\mathcal{L}_{\mathrm{BSM}}$ may contain many new sources of $C P$ violation. However, if the new states are sufficiently heavy, experiments will only be sensitive to a finite number of (linear combinations of) BSM phases. To leading order in momentum counting, the experimentally measurable phases are

\footnotetext{
${ }^{4}$ Our conventions used in this section are collected in an Appendix.
} 
encoded in the complex Wilson coefficients of the dimension 6 SMEFT operators upon matching, ${ }^{5}$

$$
\mathcal{L}^{\text {low energy }} \longrightarrow \mathcal{L}_{\mathrm{SM}}^{\prime}+\frac{1}{\Lambda^{2}} \sum_{i} c_{i} \mathcal{O}_{i}
$$

Here $\Lambda$ is the mass scale of the heavy new physics, and the Wilson coefficients $c_{i}$ introduce up to 1149 physical phases [47], assuming conservation of baryon number. The prime of $\mathcal{L}_{\mathrm{SM}}^{\prime}$ denotes the presence of corrections to the SM parameters induced by matching and renormalization effects. In particular, the strong $C P$ phase is shifted by

$$
\begin{aligned}
\delta \bar{\theta} & =\delta \theta+\delta\left(\arg \operatorname{det} Y_{u}\right)+\delta\left(\arg \operatorname{det} Y_{d}\right) \\
& \approx \delta \theta+\operatorname{Im} \operatorname{Tr}\left(Y_{u}^{-1} \delta Y_{u}\right)+\operatorname{Im} \operatorname{Tr}\left(Y_{d}^{-1} \delta Y_{d}\right)
\end{aligned}
$$

where in the second line we have expanded to first order in the threshold corrections $\delta Y_{u}$ and $\delta Y_{d}$.

Absent specific knowledge of the form of $\mathcal{L}_{\mathrm{BSM}}$, $\left\{\delta \theta, \delta Y_{u}, \delta Y_{d}\right\}$ are incalculable. However, one can estimate their natural size from SMEFT loops. Just as quadratic divergences in the Higgs mass from loops of SM fields
TABLE I. The dimension 6 operators of the standard model (in the basis of Ref. [49]) which contribute to the one loop quadratic divergence in $\bar{\theta} . \epsilon^{12}=\epsilon_{12}=+1$ and $\sigma^{\mu \nu}=\frac{1}{2} i\left[\gamma^{\mu}, \gamma^{\nu}\right]$.

\begin{tabular}{lccc}
\hline \hline $\mathcal{O}_{u H}$ & $H^{\dagger} H \overline{Q_{L i}} \tilde{H} u_{R j}$ & $\mathcal{O}_{d H}$ & $H^{\dagger} H \overline{Q_{L i}} H d_{R j}$ \\
$\mathcal{O}_{d G}$ & $\overline{Q_{L i}} \sigma^{\mu \nu} T^{a} d_{R j} H G_{\mu \nu}^{a}$ & $\mathcal{O}_{d W}$ & $\overline{Q_{L i}} \sigma^{\mu \nu} d_{R j} \tau^{a} H W_{\mu \nu}^{a}$ \\
$\mathcal{O}_{d B}$ & $\overline{Q_{L i}} \sigma^{\mu \nu} d_{R j} H B_{\mu \nu}$ & $\mathcal{O}_{u G}$ & $\overline{Q_{L L}} \sigma^{\mu \nu} T^{a} u_{R R} \tilde{H} G_{\mu \nu}^{a}$ \\
$\mathcal{O}_{u W}$ & $\overline{Q_{L i}} \sigma^{\mu \nu} u_{R j} \tau^{a} \tilde{H} W_{\mu \nu}^{a}$ & $\mathcal{O}_{u B}$ & $\overline{Q_{L i}} \sigma^{\mu \nu} u_{R j} \tilde{H} B_{\mu \nu}$ \\
$\mathcal{O}_{H u d}$ & $i \tilde{H}^{\dagger} D_{\mu} H \overline{u_{R i}} \gamma^{\mu} d_{R j}$ & $\mathcal{O}_{q u q d(1)}$ & $\epsilon^{e f} \overline{Q_{L i}^{e}} u_{R j} \overline{Q_{L k}^{f}} d_{R l}$ \\
$\mathcal{O}_{q u q d(8)}$ & $\epsilon^{e f} \overline{Q_{L i}^{e}} T^{a} u_{R j} \overline{Q_{L k}^{f}} T^{a} d_{R l}$ & $\mathcal{O}_{\text {lequ(1) }}$ & $\epsilon^{e f} \overline{L_{L i}^{e}} e_{R j} \overline{Q_{L k}^{f}} u_{R l}$ \\
$\mathcal{O}_{l e d q}$ & $\overline{L_{L i}} e_{R j} \overline{d_{R k} Q_{L l}}$ & $\mathcal{O}_{H \tilde{G}}$ & $H^{\dagger} H G_{\mu \nu}^{a} \tilde{G}^{a \mu \nu}$ \\
$\mathcal{O}_{\tilde{G}}$ & $f^{a b c} G^{a j}{ }_{\nu} G^{b \nu}{ }_{\rho} \tilde{G}^{c \rho}$ & & \\
\hline \hline
\end{tabular}

signals strong sensitivity of $m_{H}^{2}$ to UV threshold corrections, quadratically divergent corrections to $\theta$ and the quark Yukawas in SMEFT are a proxy for the threshold corrections received by these parameters at the cutoff. The one loop quadratically sensitive corrections to the SM parameters are

$$
\begin{aligned}
&\left(\delta Y_{d}\right)^{i j} \sim \frac{1}{16 \pi^{2} \Lambda^{2}}\left(3 c_{d H}^{i j}-\left(c_{H q(1)}^{i k}+3 c_{H q(3)}^{i k}\right) Y_{d}^{k j}+Y_{d}^{i k} c_{H d}^{k j}-Y_{u}^{i k} c_{H u d}^{k j}+4\left(c_{q d(1)}^{j m n i}+\frac{4}{3} c_{q d(8)}^{j m n i}\right) Y_{d}^{m n}-2 c_{l e d q}^{* m n j i} Y_{e}^{m n}\right. \\
&\left.+\left(6 c_{q u q d(1)}^{m n n i j}+c_{q u q d(1)}^{i n m j}+\frac{4}{3} c_{q u q d(8)}^{i n m j}\right) Y_{u}^{\dagger n m}+g^{\prime} c_{d B}^{i j}-18 g c_{d W}^{i j}-16 g_{s} c_{d G}^{i j}\right) \Lambda^{2} \\
&\left(\delta Y_{u}\right)^{i j} \sim \frac{1}{16 \pi^{2} \Lambda^{2}}\left(3 c_{u H}^{i j}+\left(c_{H q(1)}^{i k}-3 c_{H q(3)}^{i k}\right) Y_{u}^{k j}-Y_{u}^{i k} c_{H u}^{k j}+Y_{d}^{i k} c_{H u d}^{* j k}\right. \\
&+4\left(c_{q u(1)}^{j m n i}+\frac{4}{3} c_{q u(8)}^{j m n i}\right) Y_{u}^{m n}+2 c_{l e q u(1)}^{m n i j} Y_{e}^{\dagger n m}+\left(6 c_{q u q d(1)}^{i j m n}+c_{q u q d(1)}^{m j i n}+\frac{4}{3} c_{q u q d(8)}^{m j i n}\right) Y_{d}^{\dagger n m} \\
&\left.-5 g^{\prime} c_{u B}^{i j}-18 g c_{u W}^{i j}-16 g_{s} c_{u G}^{i j}\right) \Lambda^{2} .
\end{aligned}
$$

The $c$ s are the dimensionless Wilson coefficients of dimension 6 operators, including a variety of electromagnetic, weak, and chromo EDMs, four-fermi operators, the Weinberg operator, and various $d=4$ operators with $H^{\dagger} H$ attached. The overall correction to $\bar{\theta}$ is

$$
\begin{aligned}
16 \pi^{2} \delta \bar{\theta} \sim & 16 \pi^{2}\left(\frac{2}{g_{s}^{2}} c_{H \tilde{G}}-\frac{9}{2 g_{s}} c_{\tilde{G}}\right)+\operatorname{Im} \operatorname{Tr}\left[Y_{d}^{-1}\left(3 c_{d H}+g^{\prime} c_{d B}-18 g c_{d W}-16 g_{s} c_{d G}\right)\right] \\
& +\operatorname{Im} \operatorname{Tr}\left[Y_{u}^{-1}\left(3 c_{u H}-5 g^{\prime} c_{u B}-18 g c_{u W}-16 g_{s} c_{u G}\right)\right]+\operatorname{Im} \operatorname{Tr}\left[\left(Y_{d}^{-1} Y_{u}+Y_{d}^{\dagger}\left(Y_{u}^{\dagger}\right)^{-1}\right) c_{H u d}\right] \\
& +\operatorname{Im}\left[2 c_{l e q u(1)}^{m n i j} Y_{e}^{\dagger n m}\left(Y_{u}^{-1}\right)^{j i}-2 c_{l e d q}^{* m n i j} Y_{e}^{m n}\left(Y_{d}^{-1}\right)^{i j}\right] \\
& +\operatorname{Im}\left[\left(6 c_{q u q d(1)}^{m n i j}+c_{q u q d(1)}^{i n m j}+\frac{4}{3} c_{q u q d(8)}^{i n m j}\right)\left(Y_{u}^{\dagger n m}\left(Y_{d}^{-1}\right)^{j i}+Y_{d}^{\dagger j i}\left(Y_{u}^{-1}\right)^{n m}\right)\right] .
\end{aligned}
$$

\footnotetext{
${ }^{5}$ The dimension 5 Weinberg operator is not shown, as it will play no role in the following discussion.
} 

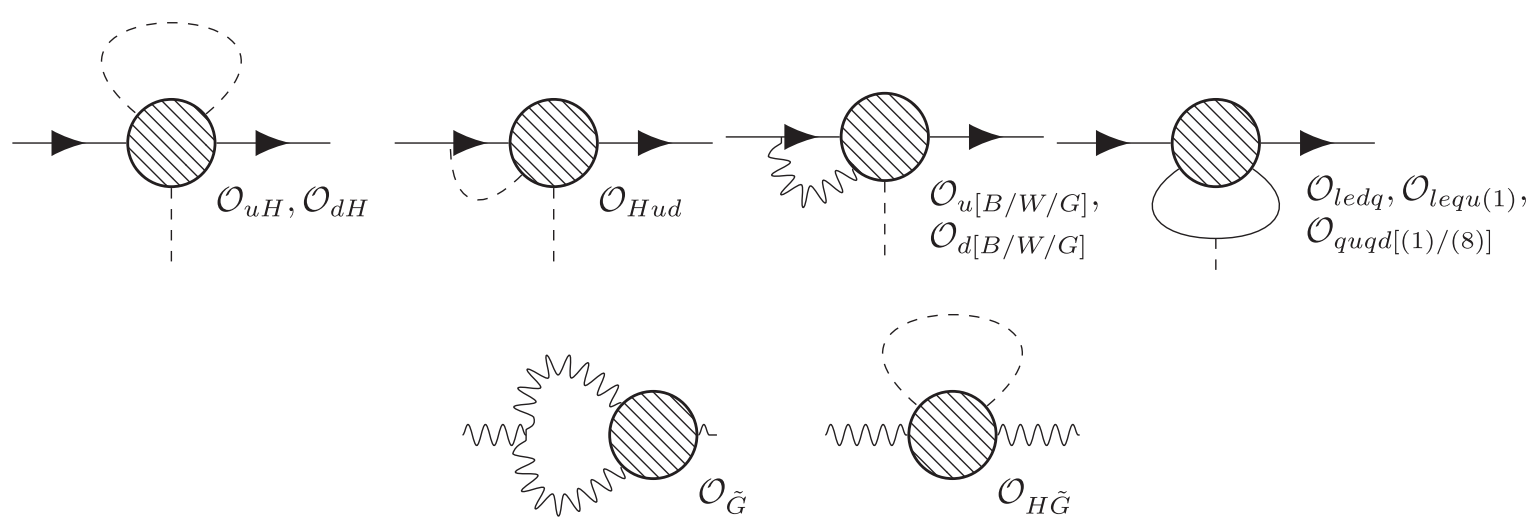

FIG. 1. Diagrammatic examples of the one-loop contributions of the dimension 6 operators (indicated by the hatched circle) to the dimension 4 strong $C P$ phase.

The contributions of $c_{H q(1)}, c_{H q(3)}, c_{H u}, c_{H d}, c_{q u(1)}, c_{q u(8)}$, $c_{q d(1)}, c_{q d(8)}$, which appear in $\delta Y_{u, d}$, vanish identically in $\delta \bar{\theta}$ due to Hermiticity. The operators whose coefficients appear explicitly in Eq. (9) are listed in Table I, and a sampling of the diagrams are shown in Fig. 1. All the loop integrals were regulated with a momentum space cutoff $\Lambda$. In the case of the Weinberg $\left(\mathcal{O}_{\tilde{G}}\right)$ and dipole operators $\left(\mathcal{O}_{[u / d][B / W / G]}\right)$, the loop integrals are scaleless; for the remaining operators, we have checked our results against the dimensionally regularized RG equations of [48].
Absent an infrared relaxation of $\bar{\theta}$, e.g. by the PecceiQuinn mechanism, naturalness requires $|\delta \bar{\theta}| \lesssim 10^{-10}$, implying a stringent bound on the combination of Wilson coefficients in (9).

We see that $\delta \bar{\theta}$ receives contributions from a considerable variety of operators, all containing colored particles but many also containing leptons and electroweak bosons. If one assumes the Wilson coefficients are minimally flavor-violating [50], i.e., at leading order in the Yukawas

$$
\begin{aligned}
c_{d[H / B / W / G]}^{i j} & =\hat{c}_{d[H / B / W / G]} Y_{d}^{i j} ; \quad c_{u[H / B / W / G]}^{i j}=\hat{c}_{u[H / B / W / G]} Y_{u}^{i j} ; \quad c_{H u d}^{i j}=\hat{c}_{H u d}\left(Y_{u}^{\dagger} Y_{d}\right)^{i j} ; \\
c_{l e q u(1)}^{i j k l} & =\hat{c}_{l e q u(1)} Y_{e}^{i j} Y_{u}^{k l} ; \quad c_{l e d q}^{i j k l}=\hat{c}_{l e d q} Y_{e}^{i j}\left(Y_{d}^{\dagger}\right)^{k l} ; \\
c_{q u q d[(1) /(8)]}^{i j k l} & =\hat{c}_{q u q d[(1) /(8)]}^{A} Y_{u}^{i j} Y_{d}^{k l}+\hat{c}_{q u q d[(1) /(8)]}^{B} Y_{u}^{k j} Y_{d}^{i l},
\end{aligned}
$$

then Eq. (9) reduces to a sum over the hatted flavor-blind phases

$$
\begin{aligned}
\left.16 \pi^{2} \delta \bar{\theta}\right|_{\mathrm{MFV}} \sim & 16 \pi^{2}\left(2 c_{H \tilde{G}}+9 g_{s} c_{\tilde{G}}\right)+3 \operatorname{Im}\left[3 \hat{c}_{d H}+g^{\prime} \hat{c}_{d B}-18 g \hat{c}_{d W}-16 g_{s} \hat{c}_{d G}+3 \hat{c}_{u H}-5 g^{\prime} \hat{c}_{u B}-18 g \hat{c}_{u W}-16 g_{s} \hat{c}_{u G}\right] \\
& +\left(\operatorname{Tr}\left[Y_{u} Y_{u}^{\dagger}\right]+\operatorname{Tr}\left[Y_{d} Y_{d}^{\dagger}\right]\right) \operatorname{Im}\left[7 \hat{c}_{q u q d(1)}^{A}+7 \hat{c}_{q u q d(1)}^{B}+7 \hat{c}_{q u q d(8)}^{A}+7 \hat{c}_{q u q d(8)}^{B}-\hat{c}_{H u d}\right] \\
& +\operatorname{Tr}\left[Y_{e} Y_{e}^{\dagger}\right] \operatorname{Im}\left[2 \hat{c}_{l e d q}+2 \hat{c}_{l e q u(1)}\right] .
\end{aligned}
$$

However, if the new physics has a different flavor structure, it need not even be $C P$ violating to give a sizable contribution to $\delta \bar{\theta}$ : the presence of the CKM phase in (9) will often suffice.

Why might this bound on the Wilson coefficients be satisfied? One, the BSM physics may couple extremely weakly to the SM, either through small couplings or through suppression by a large number of loop factors. For example, requiring that the Wilson coefficients be suppressed by a factor of

$$
\left(\frac{1}{16 \pi^{2}}\right)^{n} \sim\left(\frac{g^{\prime 2}}{16 \pi^{2}}\right)^{n^{\prime}} \sim 10^{-10}
$$

implies loop orders of $n \sim 4.5$ and $n^{\prime} \sim 3.1$. However, in this case, other effects from BSM physics would also be too small to observe.

Another possibility is that the Wilson coefficients may be of natural size, but their combination in Eq. (9) is very small (analogous to a Veltman condition for the Higgs mass.) 
This apparent fine tuning would still require explanation, especially as it is not stable under the 1-loop SMEFT RGEs [Eq. (9) is not an eigendirection of the anomalous dimension matrix of the dimension 6 operators] [47]. This RGE instability also highlights another problem: there are twoloop and higher corrections to $\bar{\theta}$ from other dimension 6 , and higher, operators which we have neglected, and may yet be significant as well. ${ }^{6}$

Thus, even if BSM physics is too heavy to be produced on-shell, observation of a nonzero SMEFT coefficient in Eq. (9) would cause significant tension for UV solutions to the strong $C P$ problem. In the following sections, we discuss sensitivities of low-energy and collider experiments to some representative operators.

\section{LOW-ENERGY PROBES}

The main observational consequence of a nonzero $\bar{\theta}$ term is the presence of nonzero EDMs of nucleons, nuclei, atoms, and molecules. The absence of a signal in all neutron-EDM experiments thus far provides the limit $\bar{\theta} \lesssim$ $10^{-10}$ and establishes the strong $C P$ problem. A signal in any of the next-generation EDM experiments, however, might point toward a finite $\bar{\theta}$ term, higher-dimensional BSM operators, or a combination of both. ${ }^{7}$ Strategies to isolate the source of $C P$ violation from multiple nonzero EDM measurements have appeared in the literature [51,52]. In particular, a scenario with a pure $\bar{\theta}$ term would lead to a rather distinct pattern of nucleon, nuclear, and diamagnetic EDMs (from now on, we will refer to these as 'hadronic' EDMs) [53,54], while lepton and paramagnetic EDMs, that are dominated by (semi-)leptonic sources of $C P$ violation, should be much smaller $[55,56]$. While it is challenging to completely isolate the source of a new observation of hadronic $C P$ violation, it is potentially easier to rule out a pure $\bar{\theta}$ scenario. As discussed above, such a result can provide indirect evidence that $\bar{\theta}$ is relaxed by the PQ mechanism. ${ }^{8}$

\footnotetext{
${ }^{6}$ For one example, the $\mathcal{O}_{\text {lequ }}^{(3)}$ operator does not appear in Table I, but it generates $\mathcal{O}_{\text {lequ }}^{(1)}$ under RG, which does appear in the table. We will comment further on $\mathcal{O}_{\text {lequ }}^{(1,3)}$ in Sec. III.

${ }^{7}$ The CKM phase contributes to EDMs at a level significantly below current and expected future experimental sensitivities, and can be neglected.

${ }^{8}$ Unfortunately, discovering EDMs consistent with $\bar{\theta}$ would provide less information. For example, it would not rule out the PQ mechanism, since, as discussed in the introduction, some level of explicit PQ-violation is expected from UV sources. Furthermore, in the presence of higher-dimensional BSM sources of $C P$ violation, such as quark chromo-EDMs [57] or certain $C P$-odd four-quark operators [58], the PQ mechanism does not relax $\bar{\theta}$ to zero, but instead to a finite value proportional to the Wilson coefficients of the BSM operators. Depending on the details of the setup, the induced $\bar{\theta}$ term can potentially dominate hadronic EDMs. See, e.g., Ref. [59] for an explicit realization in a left-right symmetric model.
}

\section{A. $\bar{\theta}$ dependence of EDMs}

In testing the consistency of putative EDM signals with the $\mathrm{SM}+$ nonzero $\bar{\theta}$, there are theoretical challenges. Nonperturbative QCD and nuclear- and atomic-structure calculations are required to link $\bar{\theta}$ to EDMs of nucleons, nuclei, and diamagnetic atoms. Nevertheless, in recent years much progress has been made which we summarize here.

Historically the most important EDM is that of the neutron. $d_{n}$ has been the target of many experiments for over six decades, leading to the present limit $\left|d_{n}\right|<3.0 \times$ $10^{-13}$ efm [3,4]. The first genuine calculation of the neutron EDM in terms of $\bar{\theta}$ was performed in Ref. [2] using current algebra techniques and coincides with a leading-order calculation in chiral perturbation theory $(\chi \mathrm{PT})$ [60]. At next-to-leading order in $\chi \mathrm{PT}$ the neutron $\mathrm{EDM}$ is given by [61]

$$
d_{n}=\bar{d}_{n}-\frac{e g_{A} \bar{g}_{0}}{8 \pi^{2} F_{\pi}}\left(\ln \frac{m_{\pi}^{2}}{m_{N}^{2}}-\frac{\pi m_{\pi}}{2 m_{N}}\right),
$$

in terms of $g_{A} \simeq 1.27$, the strong pion-nucleon axial coupling, $F_{\pi} \simeq 92.2 \mathrm{MeV}$, the pion decay constant, $m_{\pi}$, the pion mass, and two low-energy constants (LECs), $\bar{d}_{n}$ and $\bar{g}_{0}$, associated to $C P$-violating hadronic interactions that are introduced below. The expression for the proton EDM is, not surprisingly, very similar,

$d_{p}=\bar{d}_{p}+\frac{e g_{A}}{8 \pi^{2} F_{\pi}}\left[\bar{g}_{0}\left(\ln \frac{m_{\pi}^{2}}{m_{N}^{2}}-\frac{2 \pi m_{\pi}}{m_{N}}\right)-\bar{g}_{1} \frac{\pi m_{\pi}}{2 m_{N}}\right]$,

and depends on two additional LECs $\bar{d}_{p}$ and $\bar{g}_{1}$.

The LECs $\bar{g}_{0}$ and $\bar{g}_{1}$ are the coupling constants of $C P$-violating pion-nucleon interactions

$$
\mathcal{L}_{\pi N}=\bar{g}_{0} \bar{N} \vec{\tau} \cdot \vec{\pi} N+\bar{g}_{1} \bar{N} \pi_{3} N,
$$

in terms of the nucleon doublet $N=(p n)^{T}$ and pion triplet $\vec{\pi}$. The logarithm in brackets in Eqs. (13) and (14) arise from one-loop diagrams involving one insertion of $\bar{g}_{0}$, one insertion of the strong pion-nucleon coupling $g_{A}$, and a photon coupling to the pion-in-flight. This loop is divergent and the divergence and associated scale dependence is absorbed into the counterterms $\bar{d}_{n}$ and $\bar{d}_{p}$, which reflect short-distance (of distance shorter than $\sim m_{\pi}^{-1}$ ) contributions to the nucleon EDMs. The other pieces in brackets arise from finite loops at next-to-leading order in the chiral expansion.

The above $C P$-odd hadronic interactions $d_{n}, d_{p}, \bar{g}_{0}$, and $\bar{g}_{1}$ also determine the EDMs of light nuclei and diamagnetic atoms in the pure $\bar{\theta}$ scenario. Other interactions, such as short-range $C P$-odd nucleon-nucleon couplings, only appear at next-to-next-to-leading order in the chiral expansion and are expected to contribute at the $10 \%$ level $[62,63]$. 
So far there exist no EDM experiments involving light nuclei or light atoms. Light nuclei have not been used because they are charged, and standard EDM experiments apply a large electric field which would eject the nucleus from the apparatus. Light atoms are not used because Schiff's theorem [64] ensures that the EDM of a nucleus is screened inside a neutral system such as an atom. Schiff's theorem is not exact and assumes pointlike particles, but provides a very good approximation for small systems. As such, light atoms are not appropriate targets for EDM searches and much heavier systems are used.

The current best limit on any EDM is that of the ${ }^{199} \mathrm{Hg}$ atom: $d_{\mathrm{Hg}}<6.2 \times 10^{-17} e \mathrm{fm}$ [65]. Unfortunately, it is not an easy task to calculate the atomic EDM of such a complex system in terms of the above $C P$-odd interactions. Technically, it requires a calculation of the so-called nuclear Schiff moment and an atomic calculation linking the Schiff moment to the atomic EDM. At present, the atomic calculation is under relatively good control $[66,67]$, but the nuclear calculation is problematic (see Refs. $[66,68]$ for more details). The present estimate is

$$
\begin{aligned}
d_{\mathrm{Hg}}= & -(1.8 \pm 0.3) \times 10^{-4}\left[(1.9 \pm 0.1) d_{n}+(0.20 \pm 0.06) d_{p}\right. \\
& \left.+\left(0.13_{-0.07}^{+0.5} \bar{g}_{0}+0.25_{-0.63}^{+0.89} \bar{g}_{1}\right) e \mathrm{fm}\right],
\end{aligned}
$$

where the term in front of the brackets is the atomic Schiff screening factor. The main problem is the size (and even sign, in the case of $\bar{g}_{1}$ ) of the coefficients in front of the $C P$-odd pion-nucleon couplings, which are very uncertain. Advances in nuclear theory are required to improve these calculations. In specific BSM scenarios there can be other sizable contributions to $d_{\mathrm{Hg}}$, for example from (semi-) leptonic $C P$-odd interactions, but these are negligible in the pure $\bar{\theta}$ scenario. The EDM of the ${ }^{129} \mathrm{Xe}$ atom can be considered along similar lines but suffers from a larger screening factor and similar nuclear uncertainties, while the experimental limit is not as stringent [69]. Therefore we do not consider it here.

An interesting system is the ${ }^{225} \mathrm{Ra}$ atom, the EDM of which has been bounded by $d_{\mathrm{Ra}}<1.2 \times 10^{-10} e \mathrm{fm}$ [70]. While this limit is seven orders of magnitude weaker than that on $d_{\mathrm{Hg}}$, great experimental progress is expected. In addition, the atomic screening factor is less severe for this atom and, more importantly, due to its octopole-deformed shape, the coefficients in front of $\bar{g}_{0}$ and $\bar{g}_{1}$ are significantly enhanced with respect to $\mathrm{Hg}$ :

$d_{\mathrm{Ra}}=(7.7 \pm 0.8) \times 10^{-4} \cdot\left[(2.5 \pm 7.5) \bar{g}_{0}-(65 \pm 40) \bar{g}_{1}\right] e \mathrm{fm}$.

While the nuclear uncertainties are still significant, they are under relatively better control than for $\mathrm{Hg}[68,71]$.

As discussed above, EDM experiments traditionally involve neutral systems. However, it was realized that charged particles trapped in electromagnetic storage rings can also be used [72]. In this way, the g-2 collaboration set the first limit on the muon EDM [73]. Several experimental collaborations aim to construct storage rings to measure the EDMs of the proton and deuteron and perhaps even the ${ }^{3} \mathrm{He}$ nucleus. Great progress towards these measurements have been reported in Refs. $[74,75]$ and it has been claimed that an accuracy of $10^{-16} e \mathrm{fm}$ can be achieved in such a setup. While still less precise than the $d_{\mathrm{Hg}}$ measurement it must be stressed that light nuclei would not suffer from atomic screening nor from large nuclear uncertainties. These plans have lead to considerable activity in the nuclear community and the EDMs of several light nuclei have been calculated within the framework of chiral effective field theory $[53,76]$

$d_{{ }^{\mathrm{H}}}=(0.94 \pm 0.01)\left(d_{n}+d_{p}\right)+\left[(0.18 \pm 0.02) \bar{g}_{1}\right] e \mathrm{fm}$,

$$
\begin{aligned}
d_{3 \mathrm{He}}= & (0.90 \pm 0.01) d_{n}-(0.03 \pm 0.01) d_{p} \\
& +\left[(0.11 \pm 0.01) \bar{g}_{0}+(0.14 \pm 0.02) \bar{g}_{1}\right] e \mathrm{fm} .
\end{aligned}
$$

EDMs of other light nuclei such as ${ }^{6} \mathrm{Li},{ }^{9} \mathrm{Be}$, and ${ }^{13} \mathrm{C}$ have been calculated in terms of the same LECs using a nuclear cluster model [77,78]. The results indicate that such systems do not show large enhancements or suppression with respect to ${ }^{2} \mathrm{H}$ and ${ }^{3} \mathrm{He}$ EDMs.

The above relations show that we can calculate a handful of EDMs of experimental interest in terms of four hadronic $C P$-violating coupling constants. The missing link is the calculation of $d_{n}, d_{p}, \bar{g}_{0}$, and $\bar{g}_{1}$ in terms of $\bar{\theta}$. By far, the size of $\bar{g}_{0}$ is known to the highest accuracy. The $\bar{\theta}$ term can, via the axial $U(1)$ anomaly, be rotated into a complex quark mass. As such, hadronic interactions induced by $\bar{\theta}$ are linked to hadronic interactions induced by the $C P$-conserving quark mass terms [79]. This was already appreciated in Ref. [2] and $\bar{g}_{0}$ was linked to a linear combination of octet baryon masses. Recently it was realized that this relation is badly violated at higher orders in the chiral $S U(3)$ expansion and that the only reliable relation is between $\bar{g}_{0}$ and the strong proton-neutron mass splitting [80]. As the strong proton-neutron mass splitting has been a target of various lattice calculations it is known to high accuracy [81] and we obtain

$$
\bar{g}_{0}=-(14.7 \pm 2.3) \times 10^{-3} \bar{\theta} .
$$

Unfortunately a relation with comparable precision does not exist for $\bar{g}_{1}$. The main difficulty is that $\bar{g}_{1}$ is an isospinbreaking interaction while the $\bar{\theta}$ term conserves isospin. As such, $\bar{g}_{1}$ is not directly induced by $\bar{\theta}$ but only via interplay with isospin breaking via the quark masses. This obscures the link between $\bar{g}_{1}$ and the hadron mass spectrum which is

\footnotetext{
${ }^{9}$ For brevity, in what follows we refer to the ${ }^{2} \mathrm{H}$ and ${ }^{3} \mathrm{He}$ EDMs as $d_{D}$ and $d_{\mathrm{He}}$, respectively.
} 
so useful in case of $\bar{g}_{0}$. Nevertheless, a piece of $\bar{g}_{1}$ can be linked to the strong pion mass splitting. The remaining piece is unknown but has been estimated in a model in Ref. [54] where it was found to be relatively small. Adding this piece as an additional uncertainty, we obtain [80]

$$
\bar{g}_{1}=(3.4 \pm 2.4) \times 10^{-3} \bar{\theta} .
$$

The smallness of $\left|\bar{g}_{1} / \bar{g}_{0}\right|$ can be understood from the necessity of additional isospin breaking for $\bar{g}_{1}$.

Finally, we need to know the values of the nucleon EDMs. An estimate can be given by inserting the obtained values of $\bar{g}_{0}$ and $\bar{g}_{1}$ in Eqs. (13) and (14). This gives

$$
\begin{aligned}
& d_{n}=\bar{d}_{n}-(2.1 \pm 0.3) \times 10^{-3} \bar{\theta} e \mathrm{fm}, \\
& d_{p}=\bar{d}_{p}+(2.4 \pm 0.3) \times 10^{-3} \bar{\theta} e \mathrm{fm},
\end{aligned}
$$

which can be used as an estimate if it is assumed, which is often done, that the short-distance contributions $\bar{d}_{n}$ and $\bar{d}_{p}$ are small with respect to the chiral logarithm. However, chiral techniques do not allow for a solid estimate of the nucleon EDMs due to the unknown sizes of $\bar{d}_{n}$ and $\bar{d}_{p}$. Nonperturbative techniques are required. References $[82,83]$ calculated the neutron EDM directly using QCD sum rules and found

$d_{n}(\mathrm{QCD}$ sum rules $)=-(2.4 \pm 1.2) \times 10^{-3} \bar{\theta} e \mathrm{fm}$,

in reasonable agreement with the chiral estimate.

The proton EDM is expected to be of the same magnitude as the neutron EDM but with opposite sign. The sum rules analyzed in [82,83] suggest $d_{p} \approx-3 / 2 d_{n}$, while a recent calculation using a large $N_{c}$ QCD model and gauge/string duality found $d_{n}=-d_{p}=-1.8 \times 10^{-3} \bar{\theta} e \mathrm{fm}$ without an uncertainty estimate [84].

Ideally, the nucleon EDMs would be calculated with lattice QCD techniques, and in recent years several collaborations have attempted to do so [85-88]. Very accurate results at non-physical pion masses were, for example, reported in Refs. [85,87] and an extrapolation to the physical point of the data in Ref. [85] lead to $d_{n}=-(3.9 \pm 0.9) \bar{\theta} e \mathrm{fm}$. Unfortunately, it was recently argued that all existing lattice calculations suffered from spurious EDM contributions due to mixing with the $C P$-even anomalous magnetic moment [89]. Subtracting the spurious pieces lead to lattice signals consistent with zero with uncertainties larger than the model estimates given above. This implies that current lattice calculations are not yet precise enough to accurately calculate the nucleon EDMs with a small nonzero $\bar{\theta}$. Further work is required; see, for example, Refs. [90,91].

In our discussion below, we will use the QCD sum rules calculation of $d_{n}$ and set $d_{p}=-(1 \pm 0.5) d_{n}$, which essentially covers all existing estimates. However, our numerical results can also simply be regarded as an illustration, and can be straightforwardly updated without modifying the qualitative point if more precise calculations become available in the future. We express the EDMs of light nuclei and diamagnetic atoms in terms of $d_{n}, d_{p}$ and $\bar{g}_{0,1}$ via the relations given above and use Eqs. (20) and (21) to link $\bar{g}_{0,1}$ to $\bar{\theta}$.

\section{B. Excluding pure- $\overline{\boldsymbol{\theta}}$ with correlated measurements}

To rule out a pure $\bar{\theta}$ scenario, we need either a single measurement of an EDM of a leptonic or paramagnetic system which would hint at a (semi-)leptonic source of $C P$ violation, or at least two hadronic EDM measurements whose relative size is in conflict with the relations above. While (semi-)leptonic CPV would rule out a pure $\bar{\theta}$ scenario, it would not immediately point towards a PQ mechanism, since some dimension-six $C P$-violating operators involving leptons (such as the lepton EDMs themselves) do not lead to large threshold corrections to $\bar{\theta}$. We discuss paramagnetic systems further at the end of this section. Instead, we are led to consider the correlations between hadronic EDM predictions. In Fig. 2 we show contours consistent with a pure- $\bar{\theta}$ scenario for pairs of
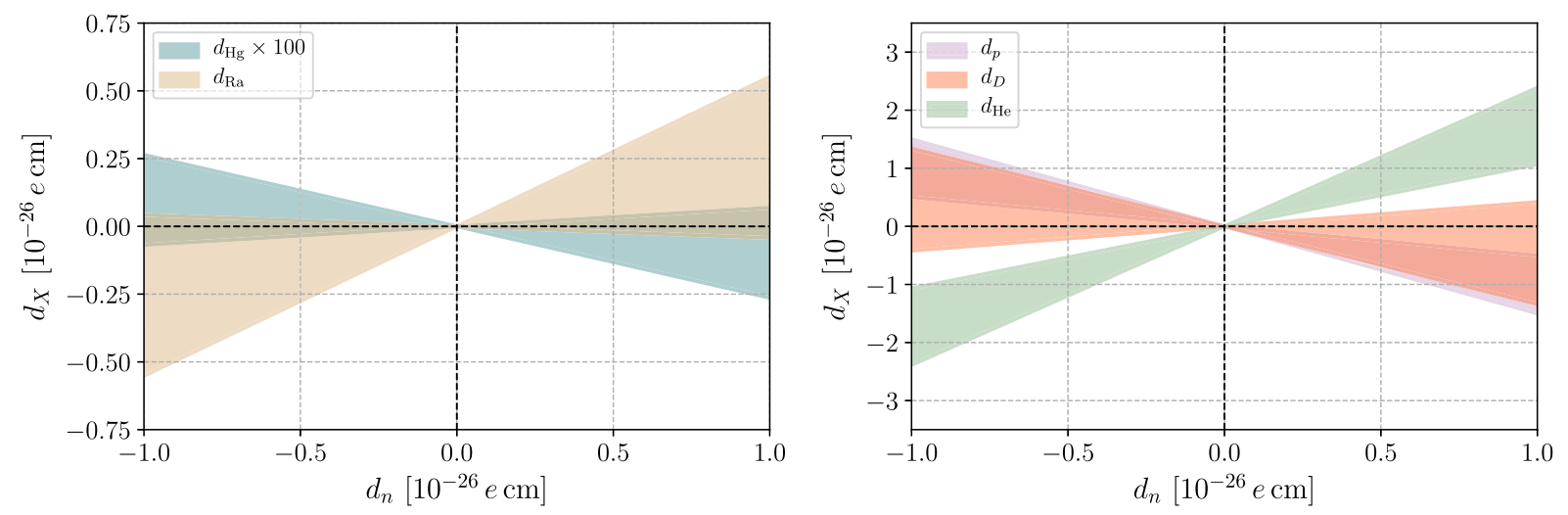

FIG. 2. Values of various EDMs as function of the neutron EDM that are consistent with a pure $\bar{\theta}$ scenario. Any EDM measurement outside of any of the shaded regions would point towards BSM sources of $C P$ violation, indicating that the strong $C P$ problem very likely requires an infrared solution. 
hypothetical hadronic EDM observations. As all EDMs depend on a single parameter, $\bar{\theta}$, all EDMs are linearly correlated, but the current theoretical uncertainties lead to contours and regions instead of lines.

The left panel of Fig. 2 shows that if $\bar{\theta}$ is the only source of $C P$ violation in these systems, the diamagnetic EDMs are expected to be small with respect to the neutron EDM due to Schiff screening (note that $d_{\mathrm{Hg}}$ has been multiplied by a factor 100 to make the contour visible). The associated uncertainties in these EDMs are also large enough that given a measurement of the neutron $\mathrm{EDM}$, the sign of $d_{\mathrm{Hg}}$ and $d_{\mathrm{Ra}}$ cannot be predicted. Nevertheless, ratios of $\left|d_{\mathrm{Ra}} / d_{n}\right| \gtrsim 1$ and $\left|d_{\mathrm{Hg}} / d_{n}\right| \gtrsim 5 \times 10^{-3}$ would point towards dimension-six sources of $C P$ violation.

How do the necessary sources compare with the operators listed in Table I, producing quadratic divergences in $\bar{\theta}$ ? The set of dimension-six operators relevant for hadronic and nuclear $C P$ violation was derived in Ref. [62], starting from the SMEFT operators and matching to a low-energy EFT around $2 \mathrm{GeV}$. At this scale, the nonleptonic operators that induce hadronic and nuclear EDMs include quark EDMs, quark chromoEDMs, the Weinberg operator, and several four-quark operators. The former three are directly induced by the operators $\mathcal{O}_{d B}, \mathcal{O}_{d W}, \mathcal{O}_{u B}, \mathcal{O}_{u W}, O_{d G}, \mathcal{O}_{u G}, \mathcal{O}_{\tilde{G}}$, all of which appear in Table I. The four-quark operators can be divided in two sets. The first set consists of operators induced by $\mathcal{O}_{\text {quqd(1) }}$ and $\mathcal{O}_{\text {quqd(8), }}$, which also appear in Table I. The final two four-quark operators do not appear in Table I as they are not $S U_{L}(2)$ gauge invariant. However, they are induced after electroweak symmetry breaking via a combination of SM weak interactions and $\mathcal{O}_{H u d}$, which does appear in Table I. Thus, deviations from the predictions of the pure $\bar{\theta}$ scenario in low-energy EDM measurements can be explained by the same SMEFT operators that point to large threshold corrections to $\bar{\theta}$.

A small aside is in order here. In principle, the $\mathrm{Hg}$ EDM, being an atomic system, gets contributions from the electron EDM and semileptonic electron-nucleon operators. The electron EDM clearly does not imply large corrections to $\bar{\theta}$. However, a nonzero $\mathrm{Hg}$ EDM in upcoming experiments would imply values of $d_{e}$ that are already ruled out by paramagnetic EDM experiments. The most relevant semileptonic electron-nucleon interaction that could induce $d_{\mathrm{Hg}}$ is the tensor operator $\bar{e} i \sigma^{\mu \nu} \gamma^{5} e \bar{N} \sigma_{\mu \nu} N$, which is mainly induced by the SMEFT operator $\epsilon^{e f} \overline{L_{L i}^{e}} \sigma^{\mu \nu} e_{R j} \overline{Q_{L k}^{f}} \sigma_{\mu \nu} u_{R l}$. While this operator does not appear in Table I, it mixes under one-loop RGE with $\mathcal{O}_{\text {lequ }}^{(1)}$, which does appear in the table. As such, values of $\left|d_{\mathrm{Hg}} / d_{n}\right|>5 \times 10^{-3}$ indeed imply dimension-six operators that induce $\bar{\theta}$ threshold corrections.
The right panel shows similar contours, but for EDMs of the light ions (proton, deuteron, and helion.) Since the theoretical control is typically better, we see clearly that $d_{p}$ and $d_{n}$ are anticorrelated, while $d_{\mathrm{He}}$ and $d_{n}$ are correlated, and $1<d_{\mathrm{He}} / d_{n}<2.5$. The deuteron EDM depends on the sum of nucleon EDMs and on $\bar{g}_{1}$, both of which are poorly known in terms of $\bar{\theta}$. As such, we cannot predict the sign of $d_{D}$ even if $d_{n}$ is known. Nevertheless we still expect $\left|d_{D}\right| \leq\left|d_{n}\right|$. Again, for many BSM sources of $C P$ violation these predictions can be quite different. In models where quark EDMs are the dominant source of $C P$ violation (for instance in splitSUSY models [92]), the neutron and proton EDM are expected to be of similar size but the relative sign can be both negative and positive. In those models, we expect $d_{\mathrm{He}} \simeq 0.9 d_{n}$ in contrast to the $\bar{\theta}$ predictions. In models with large $C P$-violating four-quark operators or chromoEDMs, the EDMs of the deuteron and helion are expected to be significantly larger than the single-nucleon EDMs due to the contributions from the $C P$-violating nuclear force induced by $\bar{g}_{0,1}$ [52].

For completeness, we briefly discuss paramagnetic systems. A nonzero EDM of a paramagnetic system would rule out a pure $\bar{\theta}$ scenario; however, it does not automatically rule out UV solutions to strong $C P$. EDMs of systems such as the $\mathrm{Tl}$ atom and $\mathrm{ThO}$ and $\mathrm{HfF}$ polar molecules are essentially dominated by two $C P$-violating effective interactions: the electron EDM and scalar electron-nucleon interactions. If the electron EDM is dominant, there is not necessarily a large threshold correction to $\bar{\theta}$. On the other hand, the semi-leptonic scalar electron-nucleon interaction arises from the $\mathcal{O}_{\text {lequ }}^{(1)}$ operator, which does generate a quadratic divergence. Recent studies [93-95] have shown that measurements of several paramagnetic systems, in addition to the diamagnetic $\mathrm{Hg} \mathrm{EDM}$, can isolate the dominant source of $C P$ violation (i.e., the electron EDM or the scalar electron-nucleon coupling). As such, even paramagnetic EDMs can in some cases provide a useful probe of radiative corrections to $\bar{\theta}$.

\section{COLLIDER PROBES}

Collider experiments are sensitive to a wide range of signatures associated with SMEFT operators. In our context, an advantage of colliders is that they provide more direct access to the individual dimension-6 CPV operators that renormalize $\bar{\theta}$ than EDM searches. On the other hand, colliders can only probe a limited subset of the operators in Table I, due to large backgrounds associated with light quark/gluon jets, and the challenge of constructing measurable $C P$-sensitive observables. Below, for illustration, we will restrict our attention to processes involving top quarks. Tops have long been recognized as offering especially promising tests of $C P$-violation beyond the standard model [96-99], since 
cross sections are large and tops can be efficiently identified and reconstructed at colliders. ${ }^{10}$

Several top quark operators appearing in Table I, including $^{11} \mathcal{O}_{t H}, \mathcal{O}_{t B}$, and $\mathcal{O}_{t W}$, contribute to the electron EDM at two loops, and are thus quite constrained by limits on $d_{e}$ [102-105], barring cancellations between contributions. In what follows we focus on the chromo-dipole moment operator $\mathcal{O}_{t G}$, since it does not contribute to the $e$-EDM at one or two loops. It can have a sizable impact on the neutron EDM $[102,104,106]$, but due to the weaker limits and significantly larger uncertainties than those associated with the $e$-EDM, it is possible for $\mathcal{O}_{t G}$ to produce observably large effects at colliders while remaining consistent with EDM bounds within their respective uncertainties.

\section{A. Top quark CEDM operator and EDM constraints}

We consider the top quark chromomagnetic and chromoelectric dipole moment operators induced by $\mathcal{O}_{t G}$ after electroweak symmetry breaking:

$$
\mathcal{L} \supset-g_{s} \frac{\tilde{\mu}_{t}}{2 m_{t}} \bar{\tau} \sigma^{\mu \nu} T^{a} t G_{\mu \nu}^{a}-i g_{s} \frac{\tilde{d}_{t}}{2 m_{t}} \bar{t} \sigma^{\mu \nu} T^{a} \gamma_{5} t G_{\mu \nu}^{a} .
$$

The chromomagnetic and chromoelectric dipole moments, $\tilde{\mu}_{t}$ and $\tilde{d}_{t}$, can be straightforwardly related to the real and imaginary parts of the corresponding Wilson coefficients before electroweak symmetry breaking:

$$
\tilde{\mu}_{t}=-\frac{2 \operatorname{Re}\left(c_{t G}\right) m_{t}^{2}}{y_{t} g_{s} \Lambda^{2}}, \quad \tilde{d}_{t}=-\frac{2 \operatorname{Im}\left(c_{t G}\right) m_{t}^{2}}{y_{t} g_{s} \Lambda^{2}} .
$$

Evidence for a nonzero $\tilde{d}_{t}$ directly implies nonzero components of $\mathcal{O}_{u G}$, and thus large threshold corrections to $\bar{\theta}$ via Eq. (9).

Due to the significant hadronic and nuclear uncertainties involved, placing robust limits on $\tilde{d}_{t}$ requires some care.

\footnotetext{
${ }^{10}$ The $C P$-odd operator $O_{H \tilde{G}}$ can also be probed at hadron colliders, for example, via angular correlations in $h+j j$ events [100]. Recently, Ref. [101] performed an analysis of this channel at the LHC and reported a $C P$-odd asymmetry in $\Delta \phi_{j j}$ of $0.3 \pm 0.2$. Assuming the significance grows in the future, Ref. [101] concluded that values of $\left|c_{H \tilde{G}} / \Lambda^{2}\right| \simeq 0.1 \mathrm{TeV}^{-2}$ can reproduce the central value. However, a study of hadronic EDMs induced by $O_{H \tilde{G}}$ concluded that $\left|c_{H \tilde{G}} / \Lambda^{2}\right|<\left(7 \times 10^{-3}\right) \mathrm{TeV}^{-2}$ from the neutron EDM limit using conservative values of the relevant matrix elements [102]. Limits on operators with Higgs fields and electroweak field strengths are even stronger because they induce the electron EDM at one loop. While EDM limits can be avoided by cancellations with other contributions, significant fine tuning (at the few-percent level) is required to align the EDM limits with the present hint of a $C P$-odd asymmetry.

${ }^{11}$ We define the third-generation operators $\mathcal{O}_{t X} \equiv \tilde{\mathcal{O}}_{u X}^{33}$, where $\tilde{\mathcal{O}}_{u X}^{i j}$ represents the operator $\mathcal{O}_{u X}^{i^{\prime} j^{\prime}}$ in Table I rotated into the quark mass basis. Here $i, j$ and $i^{\prime}, j^{\prime}$ are generation indices in the mass and gauge eigenstate bases, respectively. The corresponding Wilson coefficients $c_{t X}$ are defined analogously.
}

References $[102,104]$ performed an analysis of the constraints on $\tilde{d}_{t}$ from experimental limits on $d_{e}, d_{n}$, and $d_{\mathrm{Hg}}$ using state-of-the art matrix elements, finding an upper bound of

$$
\left|\tilde{d}_{t}\right| \lesssim 2 \times 10^{-2} \quad(90 \% \text { C.L., current }) .
$$

In this constraint, $\tilde{d}_{t}$ is evaluated at the scale ${ }^{12} \Lambda=1 \mathrm{TeV}$ and assuming that only the top CEDM operator is present at these energies. If one instead allows for the presence of other operators with comparable Wilson coefficients, the bound is weakened due to possible cancellations between contributions to the EDMs. The upper limit in Eq. (26) accounts for the various experimental uncertainties, as well as theoretical uncertainties in the predicted values of $d_{n}$ and $d_{\mathrm{Hg}}$ by varying the relevant hadronic and nuclear matrix elements across their allowed ranges. This bound therefore represents a conservative upper limit on $\tilde{d}_{t}$, allowing for possible cancellations between contributions to $d_{n}$ and/or $d_{\mathrm{Hg}}$. As a result, it is significantly weaker than results appearing elsewhere in the literature where all of the uncertainties are not accounted for in this way. If one instead adopts the central values for all matrix elements, one arrives at a significantly more stringent bound, $\left|\tilde{d}_{t}\right| \lesssim$ $1.5 \times 10^{-4}$ [104]. This dramatic difference indicates that improvements in the theoretical modeling of the neutron EDM can have a large impact on the allowed values of $\tilde{d}_{t}$. Reference [102] estimates that a robust upper limit analogous to Eq. (26) of

$$
\left|\tilde{d}_{t}\right| \lesssim 8 \times 10^{-4} \quad(90 \% \text { C.L., improved matrix elements) }
$$

can be achieved with realistic improvements in the hadronic and nuclear matrix element uncertainties. Of course more sensitive measurements will also impact these limits.

\section{B. The top CEDM and $C P$-sensitive collider observables}

Given the large uncertainties in the EDM bounds, we conservatively adopt Eq. (26) and investigate the extent to which hadron colliders can directly probe $\tilde{d}_{t}$ at this level and below. Both $\tilde{d}_{t}$ and $\tilde{\mu}_{t}$ impact various $C P$-insensitive observables at colliders, such as the Higgs and $t \bar{t}$ production rates. While many previous studies have investigated these effects [102,104,106-108], we instead focus on $C P$-odd observables sensitive to $\tilde{d}_{t}$ in the dimension-6 SMEFT, as they can provide direct evidence for a large threshold correction to $\bar{\theta}$.

\footnotetext{
${ }^{12}$ Varying the scale between $1-100 \mathrm{TeV}$ has an $\mathcal{O}(1)$ impact on the bounds, since it impacts the running of the couplings logarithmically. The bound on $\tilde{d}_{t}$ becomes weaker for $\Lambda>1 \mathrm{TeV}$.
} 
To this end, we follow Refs. [109-113] and consider $C P$-odd triple product observables in dileptonic $t \bar{t}$ production at hadron colliders (see also Ref. [114] for a study of related observables). References [111,112] showed that the expectation value of the quantity

$$
\mathcal{O}_{\mathrm{CP}} \equiv\left(\hat{\ell}_{+} \times \hat{\ell}_{-}\right) \cdot \hat{\mathbf{k}}
$$

is directly related to $\tilde{d}_{t}$. Here $\hat{\ell}_{+}$and $\hat{\ell}_{-}$are the directions of flight of the $\bar{\ell}$ and $\ell$ in the $\bar{t}$ and $t$ rest frames, respectively, and $\hat{\mathbf{k}}$ is the $t$ direction of flight in the $t \bar{t}$ center-ofmass frame. A non-zero $\left\langle\mathcal{O}_{\mathrm{CP}}\right\rangle$ results in a nonvanishing $C P$-asymmetry, $A_{\mathrm{CP}}$, defined as

$$
A_{\mathrm{CP}} \equiv \frac{N\left(\mathcal{O}_{C P}>0\right)-N\left(\mathcal{O}_{C P}<0\right)}{N\left(\mathcal{O}_{C P}>0\right)+N\left(\mathcal{O}_{C P}<0\right)}
$$

where $N$ denotes the corresponding number of dileptonic $t \bar{t}$ events. Standard model contributions to $\left\langle\mathcal{O}_{C P}\right\rangle$ are negligible, and in the operator basis used here, $\left\langle\mathcal{O}_{C P}\right\rangle$ receives a contribution only from the top chromo-EDM at leading order. Observation of $A_{C P} \neq 0$ at the LHC or a future collider would imply the need for a low-energy solution to the strong $C P$ problem.

We extend the results of Refs. [111,112] by estimating the expected sensitivity to $A_{\mathrm{CP}}$, and thus $\tilde{d}_{t}$, at the highluminosity LHC (HL-LHC) with $\sqrt{s}=14 \mathrm{TeV}$, as well as a high-energy phase of the LHC (HE-LHC) with $\sqrt{s}=$ $27 \mathrm{TeV}$ and a future $\sqrt{s}=100 \mathrm{TeV}$ collider. We account for showering/hadronization and detector resolution effects, which impact the reconstruction of the $t \bar{t}$ system necessary to determine $\mathcal{O}_{C P}$. For each center of mass energy, we used MAdGRAPH 5 [115] to generate $p p \rightarrow t \bar{t} \rightarrow$ $b \ell \bar{\nu} \bar{b} \bar{\ell}^{\prime} \nu^{\prime}$ Monte Carlo events for various values of $\tilde{d}_{t}$, utilizing a model file built by the FEYNRULES package [116]. Events were then passed to PYTHIA 6 [117] for showering/ hadronization and to DELPHES 3 [118] for fast detector simulation. For $\sqrt{s}=14,27 \mathrm{TeV}$ we use the default CMS DELPHES card with the lepton isolation criterion

$$
\sum_{i} \frac{p_{T}^{i}}{p_{T}^{\ell}}<0.1
$$

for both muons and electrons (here $p_{T}^{\ell}$ denotes the transverse momentum of the lepton or anti-lepton and $i$ denotes all other particle flow objects within a $\Delta R<0.5$ cone of $\ell$ and with $p_{T}^{i}>0.1 \mathrm{GeV}$ ). For $\sqrt{s}=100 \mathrm{TeV}$ we use the default FCC-hh detector card included in the DELPHES 3 distribution.

We select events with exactly two identified oppositelycharged leptons with $p_{T}>10 \mathrm{GeV}$ and two $b$-tagged jets, all with $|\eta|<2.5$ and $p_{T}>10 \mathrm{GeV}$. To compute $\mathcal{O}_{C P}$ for a given event, we need to reconstruct the $t \bar{t}$ system. This is nontrivial due to the two neutrinos in the final state. To do so, we take the following simple approach: we determine the neutrino four-momenta by requiring that the $\ell$ and $\bar{\nu}$ momenta reconstruct to $m_{W}$, and that the corresponding reconstructed $W$ boson and one of the $b$-jets reconstruct to the top mass, $m_{t} \approx 172 \mathrm{GeV}$. The same is required for the $\bar{\ell}$ and $\nu$ momenta and the other $b$-jet. If there are multiple real solutions for a given pairing of the $b$-jets with $W$ momenta, we choose the solution minimizing the scalar sum of the neutrino four-momenta,

$$
\sum_{i=1,2} E^{\nu_{i}}+\left|p_{x}^{\nu_{i}}\right|+\left|p_{y}^{\nu_{i}}\right|+\left|p_{z}^{\nu_{i}}\right| .
$$

In some cases, both possible pairings of $b$-jets with the reconstructed $W$ bosons yield real solutions to the equations, in which case we select the pairing with the smaller $\Delta R$ between the lepton and $b$-jet. We solve the corresponding system of equations numerically, and obtain a reconstruction efficiency of roughly $50 \%-70 \%$, depending on which numerical solver and algorithm is used. For comparison, the LHC collaborations are able to obtain up to $~ 90 \%$ reconstruction efficiencies using more sophisticated techniques (see, e.g., [113]). Thus, we expect that our sensitivity projections will be conservative from this standpoint.

With the $t \bar{t}$ system reconstructed, we compute $A_{C P}$ for each event sample. As discussed in Ref. [112], for small enough values of $\left|\tilde{d}_{t}\right|, A_{C P} \propto \tilde{d}_{t}$, since it is dominated by the interference piece between the CEDM operator and SM contribution to the $t \bar{t}$ production cross-section. We show the dependence of $A_{C P}$ on $\tilde{d}_{t}$ after our preselection cuts and reconstruction in Fig. 3 for the HL-LHC, HE-LHC, and $100 \mathrm{TeV}$ collider. Also shown are the approximate $1 \sigma$ Monte Carlo uncertainties reflecting the limited number of events generated for each point. The linear behavior is clear for all three collider energies for $\tilde{d}_{t} \lesssim 0.15$. The dashed black line in Fig. 3 corresponds to the relation

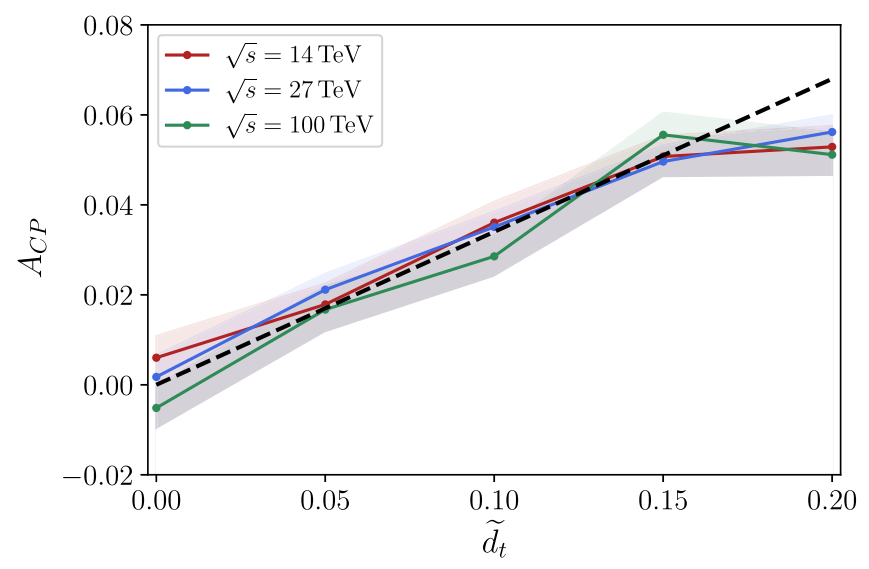

FIG. 3. Dependence of $A_{C P}$ on $\tilde{d}_{t}$ for various collider center-ofmass energies after pre-selection, reconstruction, and requiring $m_{t \bar{t}}<1 \mathrm{TeV}$. Shaded regions correspond to $1 \sigma$ Monte Carlo uncertainties. Also shown is the heuristic relation $A_{C P}=0.34 \tilde{d}_{t}$, which fits the simulated points well for $\left|\tilde{d}_{t}\right| \lesssim 0.15$, where the linear approximation begins to break down. 


$$
A_{C P} \simeq 0.34 \tilde{d}_{t}
$$

which we find to be a good match to our Monte Carlo results.

For the relatively wide range of collider center-of-mass energies we consider, it is important to ensure the validity of the EFT approach in our analysis. For all values of $\sqrt{s}$ considered, the $\tilde{d}_{t}$ contribution to the total $t \bar{t}$ cross-section is less than $50 \%$ of the SM contribution for $\left|\tilde{d}_{t}\right| \lesssim 0.15$ where the linear approximation for $A_{C P}$ holds. This suggests that the effect of the dimension-6 operators on $t \bar{t}$ production is perturbative for the momentum scales relevant for our analysis and that corrections from higherdimension operators should be under control. Furthermore, we require the $t \bar{t}$ invariant mass to satisfy $m_{t \bar{t}}<1 \mathrm{TeV}$ throughout our analysis. In particular, this requirement is reflected in the results of Fig. 3. Our results are rather insensitive to the $m_{t \bar{t}}$ cut, signaling that the effects of $\tilde{d}_{t}$ on $A_{C P}$ are dominated by events with sub-TeV momentum transfer and safely in the domain of validity of the EFT.

Our analysis neglects the effects of backgrounds mimicking dileptonic $t \bar{t}$ events. In the standard model, none of these processes contribute appreciably to the numerator of Eq. (29), but they would contribute to the denominator, and thus somewhat weaken the projected sensitivity. However, since we expect genuine $t \bar{t}$ events to strongly dominate the denominator, our projections should not be significantly affected by the inclusion of these backgrounds.

\section{Results}

Using the relation between $A_{C P}$ and $\tilde{d}_{t}$ in Eq. (32), we can estimate the sensitivity of the various colliders to $\tilde{d}_{t}$ and compare to constraints from EDM experiments. Given the standard model hypothesis, a $\sim 1 \sigma$ statistical fluctuation in the observed value of $A_{C P}$ would correspond to

$\Delta A_{C P} \simeq\left((\sigma \times \mathrm{BR}) \times \int \mathcal{L} \times\left(A \times \varepsilon \times \varepsilon_{\text {reco }}\right)\right)^{-1 / 2}$

assuming a large number of $t \bar{t}$ events so that Gaussian statistics are appropriate and that the SM contribution dominates the dileptonic $t \bar{t}$ cross-section, $\sigma \times \mathrm{BR}$. Here, $\int \mathcal{L}$ is the total integrated luminosity and $\varepsilon_{\text {reco }}$ is the efficiency for reconstructing the $t \bar{t}$ system, which we take to be $\simeq 70 \%$. $A \times \varepsilon$ is the acceptance $\times$ efficiency for identifying two oppositely charged leptons and two $b$-tagged jets meeting the kinematic requirements above at a given collider. We find $A \times \varepsilon \simeq 8 \%$ for our HL-LHC and HE-LHC analyses, while for the $100 \mathrm{TeV}$ case we find $A \times \varepsilon \simeq 15 \%$, reflecting the higher identification and tagging efficiencies in the FCC-hh DELPHES card. Requiring $A_{C P}>5 \times \Delta A_{C P}$, and using Eq. (32), we obtain $\sim 5 \sigma$ sensitivity projections for $\tilde{d}_{t}$. We find that sensitivity to

$$
\begin{aligned}
\left|\tilde{d}_{t}\right| \gtrsim 6.9 \times 10^{-3} & (\mathrm{HL}-\mathrm{LHC}) \\
3.8 \times 10^{-3} & (\mathrm{HE}-\mathrm{LHC}) \\
8.3 \times 10^{-4} & (100 \mathrm{TeV})
\end{aligned}
$$

can be reached assuming $\int \mathcal{L}=3 \mathrm{ab}^{-1}$. The reach of course improves with increased efficiencies and integrated luminosity. With $A \times \varepsilon \times \varepsilon_{\text {reco }} \simeq 20 \%$ and $\int \mathcal{L}=30 \mathrm{ab}^{-1}$, for example, our analysis suggests that a $100 \mathrm{TeV}$ collider could probe $\left|\tilde{d}_{t}\right| \gtrsim 1.9 \times 10^{-4}$. The sensitivities above only reflect statistical uncertainties; future work (and detector designs for 27 and $100 \mathrm{TeV}$ ) will be required to sharpen the above estimates by including the effects of systematic uncertainties. Our results are therefore optimistic from this standpoint.

Comparing these results with the EDM constraints on $\tilde{d}_{t}$, we see that all three colliders studied above could observe a nonzero $A_{C P}$ at the $\sim 5 \sigma$ level while remaining consistent with current EDM bounds, provided one adopts a conservative interpretation of the various uncertainties in hadronic and nuclear matrix elements. If central values are adopted, a $100 \mathrm{TeV}$ collider could still access the allowed region, provided that the neutron EDM bounds do not significantly tighten before then. In any case, cancellations between various operators could in principle allow for the HL-LHC, HE-LHC, or a $100 \mathrm{TeV}$ collider to discover a nonzero $A_{C P}$, and hence large threshold corrections to $\bar{\theta}$ while remaining consistent with improved EDM limits. Using Eq. (32), new CPV physics at scales of order 3, 5, and $10 \mathrm{TeV}$ can be probed by the HL-LHC, HE-LHC, and a future $100 \mathrm{TeV}$ collider, respectively, assuming a Wilson coefficient $c_{t G} \sim \mathcal{O}(1)$.

\section{SUMMARY}

Searches for new sources of $C P$ violation beyond the standard model are of fundamental importance, probing symmetry structure and the origin of the matter-antimatter asymmetry. We have argued that they can play an additional valuable role in discriminating how nature solves the strong $C P$ problem. Even if new physics is too heavy to be produced on-shell, if signatures of a broad class of dimension- 6 operators are experimentally observed, it will strongly disfavor models in which $\theta=0$ is an ultraviolet boundary condition and provide indirect support for the existence of a QCD axion.

Both low energy and high energy experiments are sensitive to these operators. At low energies, correlations among two or more hadronic EDM measurements can be used to reject a pure- $\theta$ explanation over a wide range of parameter space, limited primarily by theoretical uncertainties. High energy colliders can also access new $C P$-violating operators. As an example, we have analyzed the HL-LHC, HE-LHC, and $100 \mathrm{TeV}$ collider reach for a nonzero top chromo-EDM in angular observables. 
Both classes of experiments are complementary: EDM experiments offer high sensitivity, while colliders provide more direct access to individual operators, particularly in the third generation, and are insensitive to hadronic uncertainties. The insight such discoveries could provide into the resolution of the strong $C P$ problem further increases the value of these searches.

\section{ACKNOWLEDGMENTS}

We thank Hao-Lin Li, Michael Ramsey-Musolf, Adam Ritz and Felix Yu for discussions. The work of P. D. and J. K. was supported by NSF Grant No. PHY-1719642.
J. K. and D. S. gratefully acknowledge the hospitality of the Aspen Center for Physics, supported by National Science Foundation Grant No. PHY-1607611, where a portion of this work was completed. The work of K. F. was supported by Department of Energy Grant No. DE-SC0011095. The work of D.S. was supported in part by Department of Energy Grant No. DE-SC0014129.

\section{APPENDIX: CONVENTIONS}

Here we collect conventions used in the calculation of quadratic divergences in Sec. II. The standard model Lagrangian is given by

$$
\begin{aligned}
\mathcal{L}_{\mathrm{SM}}= & \sum_{F=B, W, G}-\frac{1}{4} F_{\mu \nu} F^{\mu \nu}+\sum_{\psi=Q_{L}, L_{L}, u_{R}, d_{R}, e_{R}} i \overline{i \psi^{i}} D \psi^{i}+\left|D_{\mu} H\right|^{2}-V\left(|H|^{2}\right) \\
& +\frac{\theta g_{s}^{2}}{16 \pi^{2}} G_{\mu \nu}^{a} \tilde{G}^{a \mu \nu}-\left(Y_{u}^{i j} \overline{Q_{L i}} \tilde{H} u_{R j}+Y_{d}^{i j} \overline{Q_{L i}} H d_{R j}+Y_{e}^{i j} \overline{L_{L i}} H e_{R j}+\text { H.c. }\right) .
\end{aligned}
$$

The conventions implicit in $\mathcal{L}_{\mathrm{SM}}$ align with those of [49]. To wit, we use four component spinors for the matter fields, subscripts $L$ and $R$ denoting the action of the projection operator $P_{L}=\frac{1}{2}\left(1 \mp \gamma^{5}\right)$. $\tilde{G}^{\mu \nu}=\frac{1}{2} \epsilon^{\mu \nu \alpha \beta} G_{\alpha \beta}$, where $\epsilon^{\mu \nu \alpha \beta}=-\frac{1}{4} i \operatorname{Tr}\left(\gamma^{\mu} \gamma^{\nu} \gamma^{\alpha} \gamma^{\beta} \gamma^{5}\right)$. The gauge fields are normalized such that the covariant derivative $D_{\mu} Q_{L i}=\left(\partial_{\mu}+\frac{1}{6} i g^{\prime} B_{\mu}+\right.$ $\left.\frac{1}{2} i g W_{\mu}^{i} \tau^{i}+i g_{s} G_{\mu}^{a} T^{a}\right) Q_{L i}$, where $\operatorname{Tr}\left(T^{a} T^{b}\right)=\frac{1}{2} \delta^{a b}$ and $\operatorname{Tr}\left(\tau^{i} \tau^{j}\right)=2 \delta^{i j}$, and $\left\{B_{\mu}, W_{\mu}^{i}, G_{\mu}^{a}\right\}$ are the vector potentials feeding into the field strengths via $G_{\mu \nu}^{a} \equiv \partial_{\mu} G_{\nu}^{a}-\partial_{\nu} G_{\nu}^{a}-g_{s} f^{a b c} G_{\mu}^{b} G_{\nu}^{c}$ and so forth. $\overline{Q_{L}} \tilde{H} \equiv \epsilon^{a b}{\overline{Q_{L}}}^{a} H^{b}$ in terms of weak isospin indices $a, b$, and $\epsilon^{12}=+1$.

[1] J. H. Smith, E. M. Purcell, and N. F. Ramsey, Experimental limit to the electric dipole moment of the neutron, Phys. Rev. 108, 120 (1957).

[2] R. J. Crewther, P. Di Vecchia, G. Veneziano, and E. Witten, Chiral estimate of the electric dipole moment of the neutron in quantum chromodynamics, Phys. Lett. 88B, 123 (1979); Errattum, Phys. Lett. 91B, 487(E) (1980).

[3] C. A. Baker et al., An Improved Experimental Limit on the Electric Dipole Moment of the Neutron, Phys. Rev. Lett. 97, 131801 (2006).

[4] J. M. Pendlebury et al., Revised experimental upper limit on the electric dipole moment of the neutron, Phys. Rev. D 92, 092003 (2015).

[5] L. Ubaldi, Effects of theta on the deuteron binding energy and the triple-alpha process, Phys. Rev. D 81, 025011 (2010).

[6] N. Kaloper and J. Terning, Landscaping the strong $C P$ problem, arXiv:1710.01740.

[7] M. Dine, L. Stephenson Haskins, L. Ubaldi, and D. Xu, Some remarks on anthropic approaches to the strong $C P$ problem, J. High Energy Phys. 05 (2018) 171.

[8] A. E. Nelson, Naturally weak $C P$ violation, Phys. Lett. 136B, 387 (1984).
[9] S. M. Barr, Solving the Strong CP Problem Without the Peccei-Quinn Symmetry, Phys. Rev. Lett. 53, 329 (1984).

[10] S. M. Barr, A natural class of non-Peccei-Quinn models, Phys. Rev. D 30, 1805 (1984).

[11] L. Bento, G. C. Branco, and P. A. Parada, A minimal model with natural suppression of strong $C P$ violation, Phys. Lett. B 267, 95 (1991).

[12] M. Beg and H.-S. Tsao, Strong $P, T$ Noninvariances in a Superweak Theory, Phys. Rev. Lett. 41, 278 (1978).

[13] R. N. Mohapatra and G. Senjanovic, Natural suppression of strong $p$ and $t$ noninvariance, Phys. Lett. 79B, 283 (1978).

[14] H. Georgi, A model of soft $C P$ violation, Hadronic J. 1, 155 (1978).

[15] K. S. Babu and R. N. Mohapatra, A solution to the strong CP problem without an axion, Phys. Rev. D 41, 1286 (1990).

[16] S. M. Barr, D. Chang, and G. Senjanovic, Strong CP Problem and Parity, Phys. Rev. Lett. 67, 2765 (1991).

[17] J. R. Ellis and M. K. Gaillard, Strong and weak CP violation, Nucl. Phys. B150, 141 (1979).

[18] I. B. Khriplovich, The quark electric dipole moment and the induced $\theta$-term in the Kobayashi-Maskawa model, 
Phys. Lett. B 173, 193 (1986); Yad. Fiz. 44, 1019 (1986) [Sov. J. Nucl. Phys. 44, 659 (1986)].

[19] J.-M. Grard and P. Mertens, Weakly-induced strong $C P$-violation, Phys. Lett. B 716, 316 (2012).

[20] C. Vafa and E. Witten, Parity Conservation in QCD, Phys. Rev. Lett. 53, 535 (1984).

[21] S. Aoki et al., Review of lattice results concerning lowenergy particle physics, Eur. Phys. J. C 77, 112 (2017).

[22] M. Dine, P. Draper, and G. Festuccia, Instanton effects in three flavor QCD, Phys. Rev. D 92, 054004 (2015).

[23] R. Peccei and H. R. Quinn, CP Conservation in the Presence of Instantons, Phys. Rev. Lett. 38, 1440 (1977).

[24] R. Peccei and H. R. Quinn, Constraints imposed by $C P$ conservation in the presence of instantons, Phys. Rev. D 16, 1791 (1977).

[25] S. Weinberg, A New Light Boson?, Phys. Rev. Lett. 40, 223 (1978).

[26] F. Wilczek, Problem of Strong $p$ and $t$ Invariance in the Presence of Instantons, Phys. Rev. Lett. 40, 279 (1978).

[27] H. M. Georgi, L. J. Hall, and M. B. Wise, Grand unified models with an automatic Peccei-Quinn symmetry, Nucl. Phys. B192, 409 (1981).

[28] G. Lazarides, C. Panagiotakopoulos, and Q. Shafi, Phenomenology and Cosmology with Superstrings, Phys. Rev. Lett. 56, 432 (1986).

[29] M. Kamionkowski and J. March-Russell, Planck scale physics and the Peccei-Quinn mechanism, Phys. Lett. B 282, 137 (1992).

[30] E. Witten, Some properties of O(32) superstrings, Phys. Lett. 149B, 351 (1984).

[31] T. Banks, M. Dine, and M. Graesser, Supersymmetry, axions and cosmology, Phys. Rev. D 68, 075011 (2003).

[32] M. Dine, R. G. Leigh, and A. Kagan, Supersymmetry and the Nelson-Barr mechanism, Phys. Rev. D 48, 2214 (1993).

[33] G. Hiller and M. Schmaltz, Solving the strong $C P$ problem with supersymmetry, Phys. Lett. B 514, 263 (2001).

[34] M. Dine and P. Draper, Challenges for the Nelson-Barr mechanism, J. High Energy Phys. 08 (2015) 132.

[35] P. Draper and D. McKeen, Diphotons, new vacuum angles, and strong $C P$, J. High Energy Phys. 04 (2016) 127.

[36] P. Draper, J. Kozaczuk, and J.-H. Yu, Theta in new QCDlike sectors, Phys. Rev. D 98, 015028 (2018).

[37] V. Rubakov, Grand unification and heavy axion, JETP Lett. 65, 621 (1997).

[38] P. Agrawal and K. Howe, Factoring the strong $C P$ problem, arXiv:1710.04213 [J. High Energy Phys. (to be published)].

[39] A. Albaid, M. Dine, and P. Draper, Strong $C P$ and $\mathrm{SUZ}_{2}$, J. High Energy Phys. 12 (2015) 046.

[40] T. Banks and T. J. Torres, Update on the pyramid scheme, Eur. Phys. J. C 72, 2185 (2012).

[41] M. Dine and W. Fischler, The not so harmless axion, Phys. Lett. 120B, 137 (1983).

[42] J. Preskill, M. B. Wise, and F. Wilczek, Cosmology of the invisible axion, Phys. Lett. 120B, 127 (1983).

[43] L. F. Abbott and P. Sikivie, A cosmological bound on the invisible axion, Phys. Lett. 120B, 133 (1983).

[44] N. Du et al. (ADMX Collaboration), A Search for Invisible Axion Dark Matter with the Axion Dark Matter Experiment, Phys. Rev. Lett. 120, 151301 (2018).
[45] M. B. Voloshin, On compatibility of small mass with large magnetic moment of neutrino, Yad. Fiz. 48, 804 (1988) [Sov. J. Nucl. Phys. 48, 512 (1988)].

[46] N. F. Bell, V. Cirigliano, M. J. Ramsey-Musolf, P. Vogel, and M. B. Wise, How Magnetic is the Dirac Neutrino?, Phys. Rev. Lett. 95, 151802 (2005).

[47] R. Alonso, E. E. Jenkins, A. V. Manohar, and M. Trott, Renormalization group evolution of the standard model dimension six operators III: Gauge coupling dependence and phenomenology, J. High Energy Phys. 04 (2014) 159.

[48] E. E. Jenkins, A. V. Manohar, and M. Trott, Renormalization group evolution of the standard model dimension six operators I: Formalism and lambda dependence, J. High Energy Phys. 10 (2013) 087.

[49] B. Grzadkowski, M. Iskrzynski, M. Misiak, and J. Rosiek, Dimension-six terms in the standard model Lagrangian, J. High Energy Phys. 10 (2010) 085.

[50] G. D'Ambrosio, G. F. Giudice, G. Isidori, and A. Strumia, Minimal flavor violation: An effective field theory approach, Nucl. Phys. B645, 155 (2002).

[51] O. Lebedev, K. A. Olive, M. Pospelov, and A. Ritz, Probing $C P$ violation with the deuteron electric dipole moment, Phys. Rev. D 70, 016003 (2004).

[52] W. Dekens, J. de Vries, J. Bsaisou, W. Bernreuther, C. Hanhart, U.-G. Meißner, A. Nogga, and A. Wirzba, Unraveling models of $C P$ violation through electric dipole moments of light nuclei, J. High Energy Phys. 07 (2014) 069.

[53] J. de Vries, R. Higa, C. P. Liu, E. Mereghetti, I. Stetcu, R. G. E. Timmermans, and U. van Kolck, Electric dipole moments of light nuclei from chiral effective field theory, Phys. Rev. C 84, 065501 (2011).

[54] J. Bsaisou, C. Hanhart, S. Liebig, U. G. Meißner, A. Nogga, and A. Wirzba, The electric dipole moment of the deuteron from the QCD $\theta$-term, Eur. Phys. J. A 49, 31 (2013).

[55] K. Choi and J.-y. Hong, Electron electric dipole moment and theta (QCD), Phys. Lett. B 259, 340 (1991).

[56] D. Ghosh and R. Sato, Lepton electric dipole moment and strong $C P$ violation, Phys. Lett. B 777, 335 (2018).

[57] M. Pospelov and A. Ritz, Neutron EDM from electric and chromoelectric dipole moments of quarks, Phys. Rev. D 63, 073015 (2001).

[58] V. Cirigliano, W. Dekens, J. de Vries, and E. Mereghetti, An $\epsilon^{\prime}$ improvement from right-handed currents, Phys. Lett. B 767, 1 (2017).

[59] A. Maiezza and M. Nemevšek, Strong $P$ invariance, neutron electric dipole moment, and minimal left-right parity at LHC, Phys. Rev. D 90, 095002 (2014).

[60] W. H. Hockings and U. van Kolck, The electric dipole form factor of the nucleon, Phys. Lett. B 605, 273 (2005).

[61] E. Mereghetti, J. de Vries, W. H. Hockings, C. M. Maekawa, and U. van Kolck, The electric dipole form factor of the nucleon in chiral perturbation theory to sub-leading order, Phys. Lett. B 696, 97 (2011).

[62] J. de Vries, E. Mereghetti, R. G. E. Timmermans, and U. van Kolck, The effective chiral Lagrangian from dimension-six parity and time-reversal violation, Ann. Phys. (Amsterdam) 338, 50 (2013). 
[63] J. Bsaisou, U.-G. Meißner, A. Nogga, and A. Wirzba, $P$ - and $T$-violating Lagrangians in chiral effective field theory and nuclear electric dipole moments, Ann. Phys. (Amsterdam) 359, 317 (2015).

[64] L. Schiff, Measurability of nuclear electric dipole moments, Phys. Rev. 132, 2194 (1963).

[65] B. Graner, Y. Chen, E. G. Lindahl, and B. R. Heckel, Reduced Limit on the Permanent Electric Dipole Moment of Hg199, Phys. Rev. Lett. 116, 161601 (2016); Errattum, Phys. Rev. Lett. 119, 119901(E) (2017).

[66] N. Yamanaka, B. K. Sahoo, N. Yoshinaga, T. Sato, K. Asahi, and B.P. Das, Probing exotic phenomena at the interface of nuclear and particle physics with the electric dipole moments of diamagnetic atoms: A unique window to hadronic and semi-leptonic $C P$ violation, Eur. Phys. J. A 53, 54 (2017).

[67] B. Sahoo and B. Das, Relativistic Normal Coupled-Cluster Theory for Accurate Determination of Electric Dipole Moments of Atoms: First Application to the Hg199 Atom, Phys. Rev. Lett. 120, 203001 (2018).

[68] J. Engel, M. J. Ramsey-Musolf, and U. van Kolck, Electric dipole moments of nucleons, nuclei, and atoms: The standard model and beyond, Prog. Part. Nucl. Phys. 71, 21 (2013).

[69] M. A. Rosenberry and T. E. Chupp, Atomic Electric Dipole Moment Measurement Using Spin Exchange Pumped Masers of ${ }^{129} \mathrm{Xe}$ and ${ }^{3} \mathrm{He}$, Phys. Rev. Lett. 86, 22 (2001).

[70] M. Bishof et al., Improved limit on the ${ }^{225}$ Ra electric dipole moment, Phys. Rev. C 94, 025501 (2016).

[71] J. Dobaczewski, J. Engel, M. Kortelainen, and P. Becker, Correlating Schiff Moments in the Light Actinides with Octupole Moments, Phys. Rev. Lett. 121, 232501 (2018).

[72] F. J. M. Farley, K. Jungmann, J. P. Miller, W. M. Morse, Y. F. Orlov, B. L. Roberts, Y. K. Semertzidis, A. Silenko, and E. J. Stephenson, A New Method of Measuring Electric Dipole Moments in Storage Rings, Phys. Rev. Lett. 93, 052001 (2004).

[73] G. W. Bennett et al. (Muon (g-2) Collaboration), An improved limit on the muon electric dipole moment, Phys. Rev. D 80, 052008 (2009).

[74] D. Eversmann et al. (JEDI Collaboration), New Method for a Continuous Determination of the Spin Tune in Storage Rings and Implications for Precision Experiments, Phys. Rev. Lett. 115, 094801 (2015).

[75] G. Guidoboni et al. (JEDI Collaboration), How to Reach a Thousand-Second In-Plane Polarization Lifetime with $0.97-\mathrm{GeV} / \mathrm{c}$ Deuterons in a Storage Ring, Phys. Rev. Lett. 117, 054801 (2016).

[76] J. Bsaisou, J. de Vries, C. Hanhart, S. Liebig, U.-G. Meißner, D. Minossi, A. Nogga, and A. Wirzba, Nuclear electric dipole moments in chiral effective field theory, J. High Energy Phys. 03 (2015) 104; Erratum, 05 (2015) 083.

[77] N. Yamanaka and E. Hiyama, Enhancement of the $C P$-odd effect in the nuclear electric dipole moment of ${ }^{6} \mathrm{Li}$, Phys. Rev. C 91, 054005 (2015).

[78] N. Yamanaka, Review of the electric dipole moment of light nuclei, Int. J. Mod. Phys. E 26, 1730002 (2017).

[79] E. Mereghetti, W. H. Hockings, and U. van Kolck, The effective chiral Lagrangian from the theta term, Ann. Phys. (Amsterdam) 325, 2363 (2010).
[80] J. de Vries, E. Mereghetti, and A. Walker-Loud, Baryon mass splittings and strong $C P$ violation in $\mathrm{SU}(3)$ chiral perturbation theory, Phys. Rev. C 92, 045201 (2015).

[81] D. A. Brantley, B. Joo, E. V. Mastropas, E. Mereghetti, H. Monge-Camacho, B.C. Tiburzi, and A. Walker-Loud, Strong isospin violation and chiral logarithms in the baryon spectrum, arXiv:1612.07733.

[82] M. Pospelov and A. Ritz, Theta Induced Electric Dipole Moment of the Neutron via QCD Sum Rules, Phys. Rev. Lett. 83, 2526 (1999).

[83] J. Hisano, J. Y. Lee, N. Nagata, and Y. Shimizu, Reevaluation of neutron electric dipole moment with QCD sum rules, Phys. Rev. D 85, 114044 (2012).

[84] L. Bartolini, F. Bigazzi, S. Bolognesi, A. L. Cotrone, and A. Manenti, Neutron Electric Dipole Moment from Gauge/String Duality, Phys. Rev. Lett. 118, 091601 (2017).

[85] F. K. Guo, R. Horsley, U. G. Meißner, Y. Nakamura, H. Perlt, P. E. L. Rakow, G. Schierholz, A. Schiller, and J. M. Zanotti, The Electric Dipole Moment of the Neutron from $2+1$ Flavor Lattice QCD, Phys. Rev. Lett. 115, 062001 (2015).

[86] A. Shindler, T. Luu, and J. de Vries, Nucleon electric dipole moment with the gradient flow: The theta-term contribution, Phys. Rev. D 92, 094518 (2015).

[87] C. Alexandrou, A. Athenodorou, M. Constantinou, K. Hadjiyiannakou, K. Jansen, G. Koutsou, K. Ottnad, and M. Petschlies, Neutron electric dipole moment using $N_{f}=$ $2+1+1$ twisted mass fermions, Phys. Rev. D 93, 074503 (2016).

[88] E. Shintani, T. Blum, T. Izubuchi, and A. Soni, Neutron and proton electric dipole moments from $N_{f}=2+1$ domain-wall fermion lattice QCD, Phys. Rev. D 93, 094503 (2016).

[89] M. Abramczyk, S. Aoki, T. Blum, T. Izubuchi, H. Ohki, and S. Syritsyn, Lattice calculation of electric dipole moments and form factors of the nucleon, Phys. Rev. D 96, 014501 (2017).

[90] M. Abramczyk, S. Aoki, T. Blum, T. Izubuchi, H. Ohki, and S. Syritsyn, Computing nucleon EDM on a lattice, EPJ Web Conf. 175, 06027 (2018).

[91] J. Dragos, T. Luu, A. Shindler, J. de Vries, and A. Yousif, Improvements to nucleon matrix elements within a $\theta$ vacuum from lattice QCD, in Proceedings of the 36th International Symposium on Lattice Field Theory (Lattice 2018), East Lansing, MI, United States, 2018 (2018), http://inspirehep.net/record/1693302.

[92] G. F. Giudice and A. Romanino, Electric dipole moments in split supersymmetry, Phys. Lett. B 634, 307 (2006).

[93] T. Chupp and M. Ramsey-Musolf, Electric dipole moments: A global analysis, Phys. Rev. C 91, 035502 (2015).

[94] T. Chupp, P. Fierlinger, M. Ramsey-Musolf, and J. Singh, Electric dipole moments of the atoms, molecules, nuclei and particles, arXiv:1710.02504.

[95] T. Fleig and M. Jung, Model-independent determinations of the electron EDM and the role of diamagnetic atoms, J. High Energy Phys. 07 (2018) 012.

[96] G. L. Kane, G. A. Ladinsky, and C. P. Yuan, Using the top quark for testing standard model polarization and $C P$ predictions, Phys. Rev. D 45, 124 (1992). 
[97] D. Atwood and A. Soni, Analysis for magnetic moment and electric dipole moment form-factors of the top quark via $e^{+} e^{-} \rightarrow \bar{t}$, Phys. Rev. D 45, 2405 (1992).

[98] W. Bernreuther, O. Nachtmann, P. Overmann, and T. Schrder, Angular correlations and distributions for searches of $C P$ violation in top quark production and decay, Nucl. Phys. B388, 53 (1992); Errattum, Nucl. Phys. B406, 516(E) (1993).

[99] D. Atwood, S. Bar-Shalom, G. Eilam, and A. Soni, CP violation in top physics, Phys. Rep. 347, 1 (2001).

[100] M. J. Dolan, P. Harris, M. Jankowiak, and M. Spannowsky, Constraining $\mathrm{CP}$-violating Higgs sectors at the LHC using gluon fusion, Phys. Rev. D 90, 073008 (2014).

[101] F. U. Bernlochner, C. Englert, C. Hays, K. Lohwasser, H. Mildner, A. Pilkington, D. D. Price, and M. Spannowsky, Signs of $C P$-violation in Higgs boson interactions, arXiv: 1808.06577.

[102] Y. T. Chien, V. Cirigliano, W. Dekens, J. de Vries, and E. Mereghetti, Direct and indirect constraints on $C P$-violating Higgs-quark and Higgs-gluon interactions, J. High Energy Phys. 02 (2016) 011.

[103] J. Brod, U. Haisch, and J. Zupan, Constraints on $C P$-violating Higgs couplings to the third generation, J. High Energy Phys. 11 (2013) 180.

[104] V. Cirigliano, W. Dekens, J. de Vries, and E. Mereghetti, Constraining the top-Higgs sector of the standard model effective field theory, Phys. Rev. D 94, 034031 (2016).

[105] K. Fuyuto and M. Ramsey-Musolf, Top down electroweak dipole operators, Phys. Lett. B 781, 492 (2018).

[106] J. F. Kamenik, M. Papucci, and A. Weiler, Constraining the dipole moments of the top quark, Phys. Rev. D 85, 071501 (2012); Errattum, Phys. Rev. D 88, 039903(E) (2013).

[107] A. Hayreter and G. Valencia, Constraints on anomalous color dipole operators from Higgs boson production at the LHC, Phys. Rev. D 88, 034033 (2013).

[108] J. A. Aguilar-Saavedra, B. Fuks, and M. L. Mangano, Pinning down top dipole moments with ultra-boosted tops, Phys. Rev. D 91, 094021 (2015).
[109] W. Bernreuther, A. Brandenburg, and M. Flesch, Effects of Higgs sector $C P$ violation in top quark pair production at the LHC, arXiv:hep-ph/9812387.

[110] W. Bernreuther and Z.-G. Si, Distributions and correlations for top quark pair production and decay at the Tevatron and LHC, Nucl. Phys. B837, 90 (2010).

[111] W. Bernreuther and Z.-G. Si, Top quark spin correlations and polarization at the LHC: Standard model predictions and effects of anomalous top chromo moments, Phys. Lett. B 725, 115 (2013); Errattum, Phys. Lett. B 744, 413(E) (2015).

[112] W. Bernreuther, D. Heisler, and Z.-G. Si, A set of top quark spin correlation and polarization observables for the LHC: Standard model predictions and new physics contributions, J. High Energy Phys. 12 (2015) 026.

[113] M. Aaboud et al. (ATLAS Collaboration), Measurements of top quark spin observables in $t \bar{t}$ events using dilepton final states in $\sqrt{s}=8 \mathrm{TeV}$ pp collisions with the ATLAS detector, J. High Energy Phys. 03 (2017) 113.

[114] K. Ma, Asymmetry observables for measuring spin correlations in top-quark pair production, Phys. Rev. D 96, 071501 (2017).

[115] J. Alwall, R. Frederix, S. Frixione, V. Hirschi, F. Maltoni, O. Mattelaer, H. S. Shao, T. Stelzer, P. Torrielli, and M. Zaro, The automated computation of tree-level and nextto-leading order differential cross sections, and their matching to parton shower simulations, J. High Energy Phys. 07 (2014) 079.

[116] A. Alloul, N. D. Christensen, C. Degrande, C. Duhr, and B. Fuks, FeynRules 2.0-A complete toolbox for treelevel phenomenology, Comput. Phys. Commun. 185, 2250 (2014).

[117] T. Sjostrand, S. Mrenna, and P. Z. Skands, PYTHIA 6.4 physics and manual, J. High Energy Phys. 05 (2006) 026.

[118] J. de Favereau, C. Delaere, P. Demin, A. Giammanco, V. Lematre, A. Mertens, and M. Selvaggi (DELPHES 3 Collaboration), DELPHES 3: A modular framework for fast simulation of a generic collider experiment, J. High Energy Phys. 02 (2014) 057. 